\title{
Descripción morfológica de la musculatura del miembro pélvico de un ejemplar de lechuza común (Tyto alba)
}

\author{
Morphological description of pelvic limb muscles of a specimen of barn \\ owl (Tyto alba)
}

\begin{abstract}
Pamela Morales M. ${ }^{1,2}$, Mariela Novoa G. ${ }^{1}$
El objetivo de este estudio fue describir la musculatura de los miembros pélvicos de un ejemplar de lechuza común (Tyto alba). El espécimen cuenta con músculos potentes, con predominio en el desarrollo de los músculos flexores sobre los extensores. De manera general, existe similitud con la musculatura descrita en otras aves, especialmente rapaces; sin embargo, es importante destacar algunas diferencias, como por ejemplo, el $\mathrm{m}$. iliofibular se encuentra bien desarrollado y se ubica caudal y superficial al m. iliotibial lateral; el m. femorotibial posee solo dos cabezas; los ms. ambiens y fibular largo no se observaron en el ejemplar estudiado, pero se observó el m. flexor lateral del muslo. El m. flexor digital largo posee solo una cabeza; los ms. perforantes y perforados se encuentran altamente fusionados y el $\mathrm{m}$. extensor largo del dedo I presenta una pequeña cabeza muscular.
\end{abstract}

Palabras clave: músculos, miembro pélvico, lechuza común

\section{Abstract}

The aim of this study was to describe the musculature of the pelvic limbs of a barn owl (Tyto alba). The specimen has powerful muscles, with a predominance in the development of the flexor muscles over the extensors. In general, there is similarity with the musculature described in other birds, especially raptors; however, it is important to highlight some differences, such as $\mathrm{m}$. iliofibular is well developed and is located caudal

\footnotetext{
${ }^{1}$ Escuela de Medicina Veterinaria, Facultad de Recursos Naturales y Medicina Veterinaria, Universidad Santo Tomás, Chile

${ }^{2}$ E-mail: pmoralesm@santotomas.cl
}

Recibido: 19 de enero de 2021

Aceptado para publicación: 12 de agosto de 2021

Publicado: 27 de octubre de 2021

CLos autores. Este artículo es publicado por la Rev Inv Vet Perú de la Facultad de Medicina Veterinaria, Universidad Nacional Mayor de San Marcos. Este es un artículo de acceso abierto, distribuido bajo los términos de la licencia Creative Commons Atribución 4.0 Internacional (CC BY 4.0) [https:// creativecommons.org/licenses/by/4.0/deed.es] que permite el uso, distribución y reproducción en cualquier medio, siempre que la obra original sea debidamente citada de su fuente original 
and superficial to the $\mathrm{m}$. lateral iliotibial; the $\mathrm{m}$. femorotibial has only two heads; the ms. ambiens and fibular long were not observed in the specimen studied, but $\mathrm{m}$. lateral thigh flexor was observed. The $\mathrm{m}$. long digital flexor has only one head; the ms. perforating and perforated are highly fused and $\mathrm{m}$. extensor digitorum longus I has a small muscular head.

Key word: muscles, pelvic limbs, barn owl

\section{INTRODUCCIÓN}

La lechuza común (Tyto alba) es un ave rapaz del orden Strigiformes y de la familia Tytonidae. Se encuentra desde el sur de Norteamérica hasta Chile (Alvarado et al., 2015). Se le identifica fácilmente por la forma de la cara que asemeja un corazón, además de presentar la cara y el pecho blanco (Pavéz, 2004). Esta especie pertenece al Apéndice II de la categoría de conservación (CITES), lo que implica que se encuentra en riesgo menor (BirdLife International, 2016). La caza o captura de estas lechuzas se encuentra prohibida en Chile mediante la Ley de Caza del Ministerio de Agricultura (SAG, 2015), toda vez que es un importante controlador biológico de especies que se consideran peligrosas para la salud humana (Bó et al., 2007), tales como el ratón de cola larga (Oligoryzomys longicaudatus), principal reservorio y transmisor del virus Hanta (Mills y Childs, 1998; Figueroa et al., 2007).

La lechuza común está especializada en la caza crepuscular y nocturna. Las aves rapaces se caracterizan por poseer miembros pélvicos bien desarrollados, garras potentes (Fowler et al., 2009) y un aparato músculo tendinoso que flecta la articulación tibiotarsotarsometatarsiana junto con una flexión automática de las articulaciones interfalángicas, movimiento realizado al posarse en las ramas de los árboles o cuando van a cazar alguna presa (Einoder y Richardson, 2006; Cano, 2010; Madan et al., 2017).
La mayor parte de los trabajos de investigación sobre la lechuza común se relacionan con su ecosistema y hábitos alimenticios, por lo que es importante obtener más información sobre los aspectos morfológicos, en especial de sus miembros pélvicos. Ante esto, el objetivo del presente estudio fue describir de manera morfológica y morfométrica la musculatura de los miembros pélvicos de un ejemplar de lechuza común (Tyto alba) y con esto ser un aporte en el conocimiento anatómico de esta especie silvestre protegida y beneficiosa.

\section{Materiales y Métodos}

Se utilizó un cadáver macho adulto donado por el Servicio Agrícola y Ganadero (SAG) de la Región del Maule, Chile, con una data de muerte de menos de un día. El espécimen midió $30 \mathrm{~cm}$ de largo y pesó $430 \mathrm{~g}$. La disección fue realizada en el Laboratorio de Anatomía Animal de la Carrera de Medicina Veterinaria, sede Talca, Universidad Santo Tomás.

El mismo día de su donación se realizó la fijación del cadáver mediante el uso de solución fijadora-conservadora, constituida por alcohol $96^{\circ}$, glicerina, cloruro de benzalconio, cloruro de sodio, nitrato de sodio, formalina y esencia de eucaliptus. Por tratarse de un animal de pequeño tamaño, no se realizó repleción vascular, sino que la fijación se realizó mediante la inmersión del cuerpo y se mantuvo en refrigeración por tres semanas antes de comenzar con la disección. 
Para el estudio, se retiró el tegumento para comenzar con la disección muscular de ambos miembros pélvicos. La disección se realizó desde proximal hacia distal en cada miembro (derecho e izquierdo), considerando las siguientes regiones topográficas a estudiar: región femoral, región tibiotarsal y región tarsometatarsal. En cada región, se separaron los músculos tanto por medial como por lateral, se analizaron sus tendones de origen e inserción y se realizó el reconocimiento de cada uno para su descripción y análisis morfométrico. Las mediciones individuales del largo, ancho y grosor de cada músculo fueron determinadas mediante un pie de metro (Sánchez et al., 2017). Para la descripción se utilizó la Nómina Anatómica Avium (Baumel et al., 1993). El presente estudio corresponde a un análisis de tipo descriptivo.

\section{Resultados y Discusión}

\section{Músculos de la Región Femoral}

- M. iliotibial craneal. (Figura 1). Músculo delgado ubicado en la cara craneal de la región femoral. Se origina desde la porción preacetabular de la cresta iliaca y se inserta por medial en el ligamento patelar y cresta cnemial medial. Esto es similar a lo descrito en la perdiz azul (Dendragapus obscurus) (Sisson y Grossman, 1982), en aves rapaces diurnas de las familias Falconidae y Accipitridae (Mosto, 2014) y con lo descrito para Tyto alba por Mosto (2017). Las dimensiones fueron: largo: $5.9 \mathrm{~cm}$; ancho origen: $1.6 \mathrm{~cm}$; ancho inserción: $0.6 \mathrm{~cm}$; grosor: $0.1 \mathrm{~cm}$.

- M. iliotibial lateral (Figura 1). Se sitúa caudal al $\mathrm{m}$. iliotibial craneal. Se origina en la cresta iliaca y se inserta en el ligamento patelar, similar a lo indicado por Mosto (2017). Se encuentra conformado por una cabeza de forma triangular, a diferencia de lo indicado en gallináceas donde a menudo se describen tres cabe- zas (Sisson y Grossman, 1982). En algunos ejemplares de aves rapaces diurnas se observan dos cabezas que llegan al tendón patelar (Mosto, 2014). Dimensiones: largo: $4.8 \mathrm{~cm}$; ancho origen: $1.2 \mathrm{~cm}$; ancho inserción: $0.2 \mathrm{~cm}$; grosor: $0.1 \mathrm{~cm}$.

- M. iliofibular (Figura 1). Es bien desarrollado y se ubica caudal y superficial al m. iliotibial lateral, a diferencia de lo descrito por Sisson y Grossman (1982), los cuales indican que se encuentra en profundidad y medial al $\mathrm{m}$. iliotibial lateral. Se origina en la cresta iliaca postacetabular y se inserta en la epífisis proximal de la fíbula. Dimensiones: largo: $5.5 \mathrm{~cm}$; ancho origen: $1.6 \mathrm{~cm}$; ancho inserción: $0.9 \mathrm{~cm}$; grosor: $0.3 \mathrm{~cm}$.

- M. flexor lateral del muslo o M. flexor crural lateral. (Figura 1). Posee forma triangular y se encuentra caudal al m. iliofibular. Su distribución es similar a lo descrito para la perdiz azul (Sisson y Grossman, 1982); sin embargo, difiere a lo observado por Mosto (2017) en la lechuza común, donde se observó solo el $\mathrm{m}$. flexor crural medial. Se origina desde el extremo caudal de la cresta iliaca postacetabular y el pubis y se inserta a través de un tendón que pasa entre las porciones intermedia y medial del $\mathrm{m}$. gastrocnemio para llegar al hueso tibiotarso. Dimensiones: largo: $4.7 \mathrm{~cm}$; ancho origen: $1.6 \mathrm{~cm}$; ancho inserción: $0.4 \mathrm{~cm}$; grosor: $0.4 \mathrm{~cm}$.

- M. flexor medial del muslo (Figura 1). De forma plana y acintada. Forma la parte caudomedial del muslo. Se origina de la superficie ventral del isquion y pubis y se inserta proximalmente en el hueso tibiotarso; similar a lo descrito en aves rapaces diurnas y en la lechuza (Mosto, 2014, 2017). Dimensiones: largo: $4.2 \mathrm{~cm}$; ancho: $0.6 \mathrm{~cm}$; grosor: $0.2 \mathrm{~cm}$.

- $\quad$ M. femorotibial (Figura 1). Posee solo dos cabezas, las que corresponden al $\mathrm{m}$. femorotibial intermedio y $m$. femorotibial 

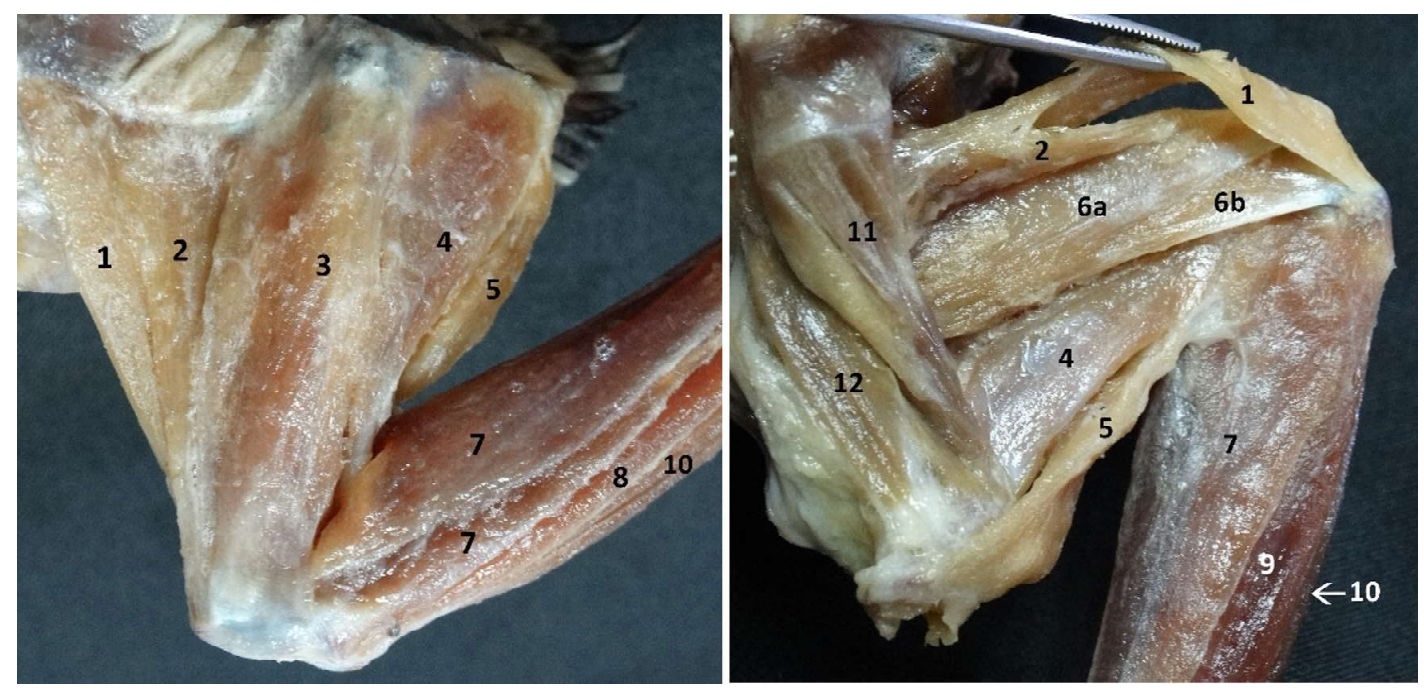

Figura 1. Miembro pélvico izquierdo de un espécimen de lechuza común (Tyto alba). Izquierda: Vista lateral de la región femoral. Derecha: Vista medial de la región femoral. 1. M. iliotibial craneal; 2. M. iliotibial lateral; 3. M. iliofibular; 4. M. flexor lateral del muslo; 5. M. flexor medial del muslo; 6a. M. femorotibial intermedio; 6b. M. femorotibial medial; 7. M. gastrocnemio; 8. M. flexor digital largo; 9. M. plantar; 10. M. tibial craneal, 11. M. iliotroncal craneal; 12. M. iliotroncal caudal

medial, lo cual difiere a lo indicado por Mosto (2017), donde se observaron tres cabezas. En algunas aves rapaces diurnas se describe una fusión entre los músculos femorotibial lateral y femorotibial intermedio (Mosto, 2014), lo que también sucede en la lechuza según Mosto (2017); sin embargo, en el espécimen estudiado las porciones se ubican y observan hacia medial. El m. femorotibial intermedio es un músculo grande en comparación con la porción medial y se origina en la cresta trocantérica craneal. El m. femorotibial medial se origina de la superficie medial del fémur, caudal a la porción intermedia. Ambas porciones forman el tendón patelar medialmente. Dimensiones: largo: $4.4 \mathrm{~cm}$; ancho: 1.1 cm; grosor: $0.4 \mathrm{~cm}$.

- M. ambiens. No se observó su presencia, aunque pudiera corresponder a fibras altamente fusionadas al $\mathrm{m}$. femo- rotibial intermedio. Esto difiere a lo indicado en otras aves (Sisson y Grossman, 1982) como también en aves rapaces diurnas, donde se indica su presencia (Mosto, 2014); sin embargo, este resultado coincide a lo descrito por Mosto (2017) para Tyto alba.

\section{Músculos de la Región Tibiotarsal}

- M. fibular largo. No se encuentra en el ejemplar estudiado. Sisson y Grossman (1982) indican que este músculo no está presente en la lechuza común; sin embargo, se encuentra en aves como pollos y pavos, ubicándose craneal al m. tibial craneal. Asimismo, se describe en algunas aves rapaces (Mosto, 2014).

- M. tibial craneal (Figuras 1, 2). Es alargado, medial y bien desarrollado. Se observaron dos cabezas bien separadas en 

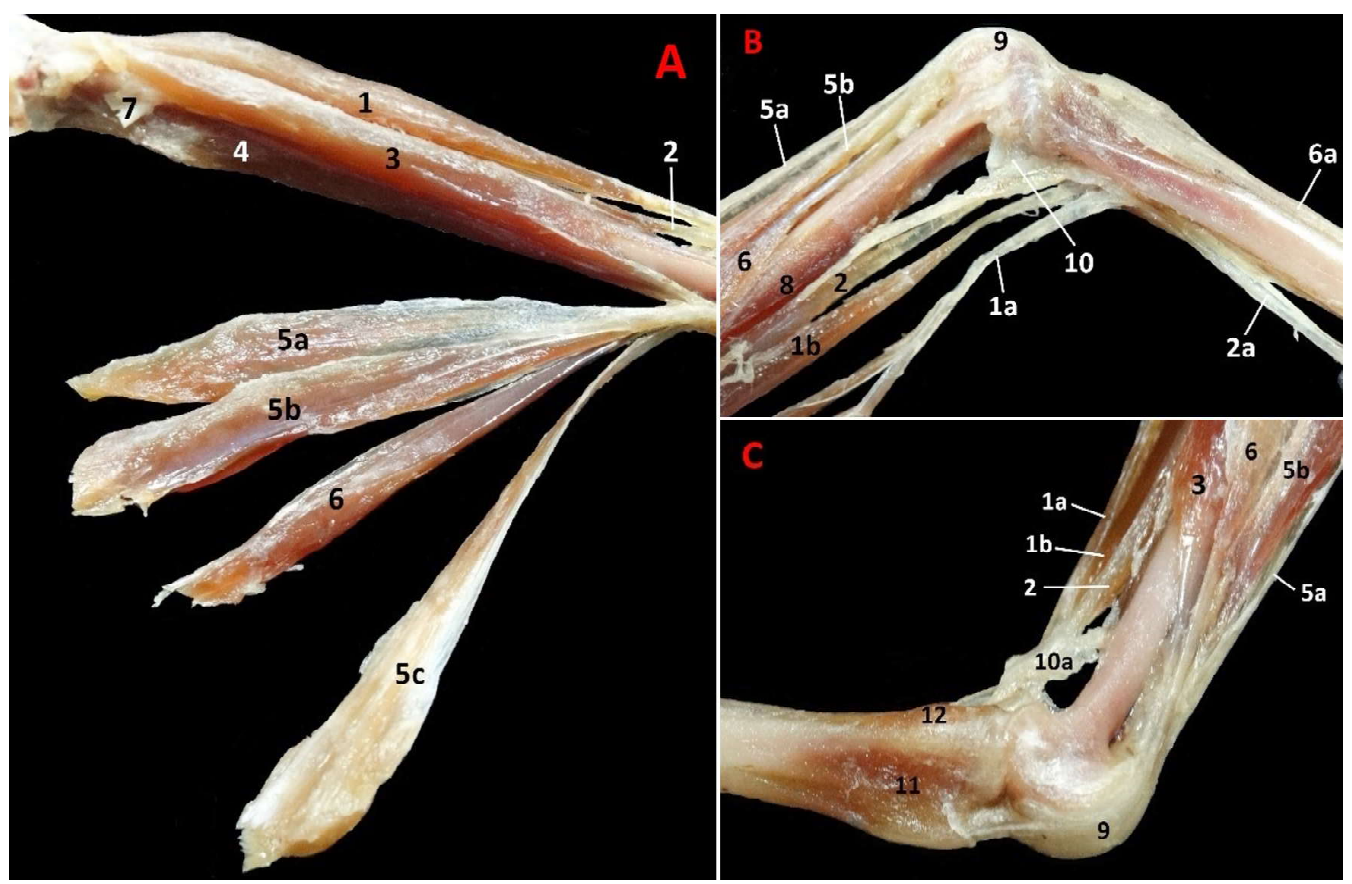

Figura 2. Región tibiotarsal y región tarsometatarsiana de un espécimen de lechuza común (Tyto $a l b a)$. A. Vista medial de la región tibiotarsiana. 1. Cabeza superficial del m. tibial craneal; 2. M. extensor digital largo; 3. M. plantar; 4. M. flexor digital largo; 5a. Cabeza lateral del $\mathrm{m}$. gastrocnemio; $5 \mathrm{~b}$. Cabeza intermedia del $\mathrm{m}$. gastrocnemio; $5 \mathrm{c}$. Cabeza medial del m. gastrocnemio; 6. M. perforados y perforantes II, III y IV; 7. Retináculo flexor. B. Vista lateral de la articulación tibiotarso-tarsometatarsiana; 1a. Cabeza superficial del $\mathrm{m}$. tibial craneal; $1 \mathrm{~b}$. Cabeza profunda del m. tibial craneal; $6 \mathrm{a}$. Tendones perforados y perforantes; 8 . M. fibular breve; 9 . Cartílago tibial (superficial a hipotarso); 10. Retináculo extensor seccionado. C. Vista lateral de la articulación tibiotarsotarsometatarsiana. 10a. Retináculo extensor; 11. M. flexor breve; 12. M. extensor largo del dedo I

todo su recorrido, una más profunda y menos desarrollada, que se origina de la cresta cnemial lateral del hueso tibiotarso, mientras que la cabeza más superficial se origina desde la cresta cnemial medial y lateral del hueso tibiotarso. Los tendones de ambas cabezas atraviesan el retináculo extensor (Figuras 2B,C) y se insertan en el surco metatarsiano del hueso tarsometatarso. Este hallazgo es similar a otras aves rapaces y aves domésticas (Sisson y Grossman; Mosto, 2014, 2017). Dimen- siones de la cabeza medial: largo: 10.5 $\mathrm{cm}$; ancho origen: $0.8 \mathrm{~cm}$; ancho unión musculotendínea: $0.2 \mathrm{~cm}$; grosor vientre muscular: $0.6 \mathrm{~cm}$. Dimensiones de la cabeza lateral: largo $9.1 \mathrm{~cm}$; ancho origen: $0.3 \mathrm{~cm}$; ancho unión musculotendínea: $0.1 \mathrm{~cm}$; grosor vientre muscular: $0.3 \mathrm{~cm}$.

- M. extensor digital largo (Figuras 1, 2) Es delgado y aplanado, cubre la superficie craneal del tibiotarso. Está profundo al $\mathrm{m}$. tibial craneal y se origina entre 
las crestas cnemiales lateral y medial, su tendón pasa a través del retináculo extensor (Figuras 2B,C) y luego se trifurca en el tarsometatarso para insertarse en el tubérculo extensor de la falange ungueal de los dedos II, III y IV. La distribución descrita es similar a otras aves rapaces (Mosto, 2014) y aves domésticas (Sisson y Grossman, 1982). Dimensiones: largo: $18.2 \mathrm{~cm}$; ancho origen: 0.7 $\mathrm{cm}$; ancho unión musculotendínea: 0.2 $\mathrm{cm}$; grosor: $0.3 \mathrm{~cm}$.

- M. fibular breve. Es pequeño y laterodistal en el tibiotarso. Se encuentra entre los músculos extensor digital largo y flexor digital largo. Se observa muy adherido al margen lateral del hueso tibiotarso. Se origina cercano al espacio interóseo y se inserta en la superficie lateral del tarsometatarso. Lo descrito es similar a otras aves domésticas (Sisson y Grossman, 1982) así como a la lechuza (Mosto, 2017). Dimensiones: largo: $6.7 \mathrm{~cm}$; ancho: $0.4 \mathrm{~cm}$. El grosor no fue posible medirlo debido a su adherencia al hueso.

- M. flexor digital largo (Figuras 1, 2). Se observó cubriendo de manera extensa la superficie caudal de la región del tibiotarso desde medial a lateral. Es profundo a la porción medial del $\mathrm{m}$. gastrocnemio. En el ejemplar estudiado se observa una sola cabeza bien desarrollada, en tanto que Mosto (2014) indica la presencia de dos cabezas musculares en otras aves rapaces. Mosto (2017) indica además, que la lechuza tendría tres cabezas musculares. Por lateral este músculo se origina de la cabeza de la fíbula y por caudal en el tibiotarso, hacia distal el tendón de inserción pasa a través del cartílago tibial, luego se trifurca y se inserta en los dedos II, III y IV. Dimensiones: largo: $15.7 \mathrm{~cm}$; ancho: $0.5 \mathrm{~cm}$; grosor: $0.3 \mathrm{~cm}$.
- M. gastrocnemio (Figuras 1, 2). Es grande y desarrollado. Se observó formado por tres cabezas, lo cual también se describe para otras aves (Sisson y Grossman, 1982; Mosto, 2014), como también se corresponde con lo observado por Mosto (2017) para la lechuza común. La porción lateral tiene su origen en la base del cóndilo lateral femoral. La porción intermedia se encuentra profunda a las otras dos porciones, estando más desarrollada que las porciones lateral y medial, tal como indica Mosto (2017) para la lechuza común; no obstante, es la menos desarrollada en algunas aves rapaces diurnas (Mosto, 2014). Su origen es en la zona poplítea y desde el cóndilo lateral de la tibia. La porción medial es aplanada y de forma triangular, se origina en la cresta cnemial medial. Todas las porciones se insertan en el hipotarso donde se observa el cartílago tibial (Figuras 2B, 2C). Dimensiones de la cabeza lateral: largo: $9.2 \mathrm{~cm}$; ancho de origen: $1.2 \mathrm{~cm}$; ancho unión musculotendínea: $0.5 \mathrm{~cm}$; grosor: $0.2 \mathrm{~cm}$. Dimensiones de la cabeza intermedia: largo: $8.7 \mathrm{~cm}$; ancho de origen: $0.7 \mathrm{~cm}$; ancho unión musculotendínea: $0.3 \mathrm{~cm}$; grosor: $0.3 \mathrm{~cm}$. Dimensiones de la cabeza medial: largo: $9.2 \mathrm{~cm}$; ancho origen: $1.5 \mathrm{~cm}$; ancho unión musculotendínea: 0.3 $\mathrm{cm}$; grosor: $0.1 \mathrm{~cm}$.

- M. plantar (Figuras 1,2) Se ubica en la cara medial del tibiotarso. Se origina en el extremo proximal del tibiotarso y se inserta en el extremo proximal del cartílago tibial. Sisson y Grossman (1982) y Mosto (2014) describen que su presencia es variada dentro de las distintas especies de aves; así, Sisson y Grossman (1982) indican que está presente en la perdiz azul, en tanto que Mosto (2014) menciona que está presente en algunas especies de aves rapaces diurnas. Dimensiones: largo: $7.9 \mathrm{~cm}$; ancho: $0.8 \mathrm{~cm}$; grosor: $0.3 \mathrm{~cm}$. 
- $\quad$ M. perforados y perforantes II, III y IV (Figura 2A) Se encuentran fusionados y profundos a la porción intermedia del músculo gastrocnemio, a diferencia de lo indicado por Sisson y Grossman (1982) y Mosto (2014) donde se describen todos de manera independiente. Se origina desde el cóndilo lateral del fémur, cabeza de la tibia y fíbula, el tendón pasa a través del cartílago tibial y el hipotarso, para insertarse en los dedos II, III y IV, respectivamente. Todos son perforados por los tendones de los músculos flexores profundos. Sisson y Grossman (1982) describen que el perforado III también esta perforado por el tendón del m. fibular largo en la perdiz azul, lo que no sucede en este ejemplar de lechuza. Dimensiones: largo: $8.4 \mathrm{~cm}$; ancho: $10.1 \mathrm{~cm}$; en tanto que el grosor no se pudo medir debido a su adherencia al hueso.

\section{Músculos de la Región Tarsometatar- siana}

- $\quad$ M. flexor breve (Figura 2C). Es poco desarrollado. Se encuentra proximal y plantar en el tarsometatarso donde se origina, su tendón de inserción es largo, ya que recorre hasta la falange proximal del dedo I. Su recorrido es similar a lo escrito por Mosto (2017), aunque de distribución más distal en el hueso tarsometatarso. Dimensiones: largo muscular: $6.8 \mathrm{~cm}$; ancho: $0.6 \mathrm{~cm}$.

- $\quad$ M. extensor largo del dedo I (Figura 2C). Es poco desarrollado y se observa una sola cabeza a diferencia de lo mencionado por Mosto (2017) que indica la presencia de dos cabezas. Se origina en la superficie dorsomedial de la epífisis proximal del tarsometatarso y se inserta en la falange ungueal del dedo I. Dimensiones: largo: $6.6 \mathrm{~cm}$; ancho: $0.6 \mathrm{~cm}$.
- M. abductor digital IV. Es muy pequeño y se ubica lateral en la epífisis proximal del tarsometatarso, similar a lo descrito por Mosto (2017). Su tendón se inserta lateral en la base de la primera falange del dedo IV. Sus dimensiones son: largo muscular: $6.3 \mathrm{~cm}$; ancho: $0.5 \mathrm{~cm}$.

En los músculos de la región tarsometatarsiana no se pudo medir el grosor debido a su adherencia al hueso.

\section{Conclusiones}

El espécimen de lechuza común (Tyto $a l b a$ ) estudiado presenta un predominio en el desarrollo de los músculos flexores sobre los extensores. Existe similitud con la morfología descrita en otras aves, principalmente rapaces, aunque es importante destacar algunas diferencias.

\section{Literatura Citada}

1. Alvarado $S$, Figueroa $R$, Valladares $P$, Carrasco-Lagos $P$, Moreno $R$. 2015. Aves rapaces de la Región Metropolitana de Santiago, Chile. Santiago: Univ. de Chile. 132 p.

2. BirdLife International. 2016. Common barn owl. Tyto alba. The IUCN Red List of Threatened Species 2016: e.T22688504A86854321. [Internet]. Available in: http://dx.doi.org/ 10.2305/IUCN.UK.2016-3.RLTS.T22688504A86854321.en

3. Bó MS, Balandrón A, Biondi LM. 2007. Ecología trófica de Falconiformes y Estringiformes: tiempo de síntesis. Hornero 22: 97-115.

4. Baumel J, King A, Breazile J, Evans H, Vanden Berge J. 1993. Handbook of avian anatomy: Nomina anatómica 
avium. $2^{\text {nd }}$ ed. Cambridge: Nuttall Ornithological Club Publication. $779 \mathrm{p}$.

5. Cano FG 2010. Anatomía específica de aves: aspectos funcionales y clínicos. España: Facultad de Veterinaria, Universidad de Murcia. $17 \mathrm{p}$.

6. Einoder L, Richardson A. 2006. An ecomorphological study of the raptorial digital tendon locking mechanism. The Ibis 148: 515-525.

7. Figueroa R, Murúa R, Schlatter R, Ruiz J, Briones M, Figueroa M, Corales E, Alvarado S. 2007. Biocontrol of hantavirus rodent reservoirs by raptorial birds in southern South America: implication for management in rural environments. In: Abstracts VII International Conference HFRS, HPS and Hantavirus, Buenos Aires, Argentina.

8. Fowler DW, Freedman EA, Scannella $J B .2009$. Predatory functional morphology in raptors: interdigital variation in talon size is related to prey restraint and immobilisation technique. PLoS One 4(11):e7999. https://doi.org/10.1371/ journal.pone.0007999

9. Madan MA, Rayfield EJ, Bright JA. 2017. Scaling and functional morphology in stringiform hind limbs. Sci Rep 7: 44920. doi: 10.1038/srep44920.

10. Mills JN, Childs JE. 1998. Ecologic studies of rodent reservoirs: their- relevance for human health. Emerg Infect Dis 4: 529-537. doi: 10.3201/ eid0404.980403

11. Mosto MC. 2014. Estructura y función del complejo apendicular posterior en aves rapaces diurnas (Falconidae y Accipitridae). Tesis Doctoral. Argentina: Univ. Nacional de la Plata. $116 \mathrm{p}$.

12. Mosto MC. 2017. The hinlimb myology of Tyto alba (Tytonidae, Strigiformes, Aves). Anat Histol Embryol 46: 25-37. doi: 10.1111/ahe.12227

13. [SAG] Servicio Agrícola y Ganadero. 2015. Especies prohibidas de caza y captura. [Internet]. Disponible en: https:// www.sag.gob.cl/ambitos-de-accion/especies-prohibidas-de-caza/145/registros

14. Sánchez J, Morales P, Medina R. 2017. Descripción anatómica de la miología del miembro pélvico y su relación topográfica con el sistema vascular y nervioso en un ejemplar de Pudu (Pudu puda). Int J Morphol 35: 1370-1376. doi: 10.4067/S0717-95022017000401370

15. Sisson S, Grossman JD. 1982. Anatomía de los animales domésticos. Tomo II. $5^{\circ}$ ed. Barcelona, España: Masson. $2302 \mathrm{p}$.

16. Pavéz EF. 2004. Descripción de las aves rapaces chilenas. En: Aves rapaces de Chile. Chile: CEA: Ed CEA. $p$ 29-104. 